\title{
Smale-Like Decomposition and Forman Theory for Discrete Scalar Fields
}

\author{
Lidija Čomić $^{1}$, Mohammed Mostefa Mesmoudi ${ }^{2}$, and Leila De Floriani ${ }^{2}$ \\ 1 Faculty of Engineering, University of Novi Sad, Serbia \\ 2 Department of Computer Science, University of Genova, Italy
}

\begin{abstract}
Forman theory, which is a discrete alternative for cell complexes to the well-known Morse theory, is currently finding several applications in areas where the data to be handled are discrete, such as image processing and computer graphics. Here, we show that a discrete scalar field $f$, defined on the vertices of a triangulated multidimensional domain $\Sigma$, and its gradient vector field Grad $f$ through the Smale-like decomposition of $f[6]$, are both the restriction of a Forman function $F$ and its gradient field Grad $F$ that extends $f$ over all the simplexes of $\Sigma$. We present an algorithm that gives an explicit construction of such an extension. Hence, the scalar field $f$ inherits the properties of Forman gradient vector fields and functions from field Grad $F$ and function $F$.
\end{abstract}

Keywords: Morse Theory, Forman Theory, Morse Decomposition.

\section{Introduction}

Morse theory is a powerful tool for understanding the topology and the geometry of a manifold $M$ on which a $C^{2}$-differentiable real-valued function $f$ is defined. A Morse function $f$ induces decompositions of $M$ (called Morse complexes) into regions associated with critical points of $f$, based on the study of the behavior of the gradient vector field of $f$. In 1998, Forman introduced a new theory for cell complexes, that is a discrete equivalent to Morse theory [8]. He proved that almost all the main results from Morse theory are valid for discrete functions.

We describe a discrete decomposition for a triangulated $n$-dimensional domain $\Sigma$ with manifold carrier, associated with a scalar field $f$ and originally proposed in [6], called a Smale-like decomposition. This decomposition simulates the Morse complexes of $f$ in the discrete case, and defines a discrete gradient field Grad $f$, which represents the topological structure of the field. We have used such decomposition in combination with simplification operations to build a multi-scale morphological representation of scalar fields [2, 5].

We construct an extended form of discrete gradient field Grad $f$ defined by a Smale-like decomposition, called an extended discrete gradient field EGrad $f$. The extended form always points in the direction in which function $f$ is descending, and thus it agrees with the (negative) flow induced by the scalar field $f$. We show that this field is a Forman gradient vector field $V_{F}$ of a Forman function $F$, whose restriction over the vertices of $\Sigma$ coincides with the initial scalar field $f$.

I. Debled-Rennesson et al. (Eds.): DGCI 2011, LNCS 6607, pp. 4774488, 2011.

(C) Springer-Verlag Berlin Heidelberg 2011 
We give the explicit formulation of a Forman function $F$ that satisfies the above property.

As a consequence, we have that it is possible to use all the machinery of Forman theory without actually working with the entire Forman function $F$, or even with the related Forman gradient vector field $V_{F}=E G r a d f$; the vector sub-field Grad $f$ is sufficient to study the behavior of scalar field $f$. Specifically, EGrad $f$ (and thus $F$ ) can be simplified by applying a cancellation operator, which eliminates critical cells of EGrad $f$ in pairs. This simplification operator will form a basis of a hierarchical representation of the morphology of the initial scalar field $f$, and of the simplicial complex $\Sigma$. We intend to build such a hierarchical model, based on a cancellation operator, in the near future.

The remainder of this paper is organized as follows. In Sections 2 and 3 . we summarize some results related to Morse and Forman theory, respectively. In Section 4, we review related work in this area. In Section 5, we recall the algorithm that computes the Smale-like decomposition. In Section 6, we present the algorithmic construction of a discrete vector field EGrad $f$, which extends the discrete gradient field Grad $f$ induced by the Smale-like decomposition to a Forman gradient vector field. In Section [7, we define a Forman function $F$, whose Forman gradient vector field $V_{F}$ coincides with EGrad $f$. In Section 8 , we draw some concluding remarks and discuss our current and future work.

\section{Morse Theory and Morse Complexes}

Morse theory captures the relationship between the topology of a manifold $M$ and the critical points of a scalar function $f$ defined on the manifold [13, 14].

Let $f$ be a $C^{2}$ real-valued function defined over a closed compact $n$-manifold $M$. A point $p$ is a critical point of $f$ if and only if the gradient $\nabla f=\left(\frac{\partial f}{\partial x_{1}}, \ldots, \frac{\partial f}{\partial x_{n}}\right)$ (in some local coordinate system around $p$ ) of $f$ vanishes at $p$. Function $f$ is a Morse function if all its critical points are non-degenerate. The number $i$ of negative eigenvalues of the Hessian matrix Hess $f$ is called the index of critical point $p$, and $p$ is called an $i$-saddle. A 0 -saddle (an $n$-saddle) is also called a minimum (a maximum). An integral line of $f$ is a maximal path which is everywhere tangent to $\nabla f$. Each integral line starts and ends at critical points of $f$, called its origin and its destination.

Integral lines that converge to (originate at) a critical point $p$ of index $i$ form an $i$-cell $((n-i)$-cell), called a descending (ascending) cell of $p$. The descending and ascending cells decompose $M$ into descending and ascending Morse complexes, denoted as $\Gamma_{d}$ and $\Gamma_{a}$, respectively. A Morse function $f$ is called a Morse-Smale function if each non-empty intersection of a descending and an ascending cell is transversal. These intersections define a Morse-Smale complex.

\section{Forman Theory}

Forman theory is a discrete counterpart of Morse theory, and its main purpose is to transpose the results of Morse theory from a smooth to a combinatorial 
setting. A function $F$, defined on all simplexes (and not only on vertices) of a finite simplicial complex $\Sigma$, is called a Forman function if for any $p$-simplex $\sigma$, all the $(p-1)$-simplexes in the boundary of $\sigma$ have a lower $F$ value than $\sigma$, and all the $(p+1)$-simplexes in the coboundary of $\sigma$ have a higher $F$ value than $\sigma$, with at most one exception. A simplex is critical if there is no exception to this rule. More formally, a function $F: \Sigma \rightarrow \mathbb{R}$ is a Forman function if for every $p$-simplex $\sigma$, both the following conditions are satisfied

$$
\text { (1) } \#\left\{\tau^{(p+1)}>\sigma: F(\tau) \leq F(\sigma)\right\} \leq 1, \quad(2) \#\left\{\nu^{(p-1)}<\sigma: F(\nu) \geq F(\sigma)\right\} \leq 1 .
$$

These inequalities cannot be equalities at the same time. A $p$-simplex $\sigma \in \Sigma$ is a critical simplex of index $p$ if both the following conditions are satisfied

(1) $\#\left\{\tau^{(p+1)}>\sigma: F(\tau) \leq F(\sigma)\right\}=0$

(2) $\#\left\{\nu^{(p-1)}<\sigma: F(\nu) \geq F(\sigma)\right\}=0$

The absolute minimum of $F$ on a a triangulation of a closed manifold occurs at a vertex; the absolute maximum occurs at a maximal dimensional simplex [8].

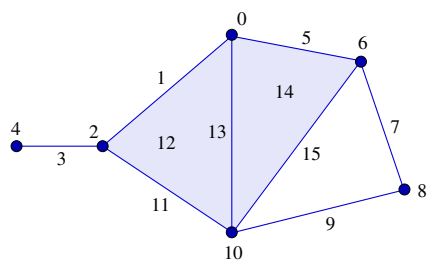

(a)

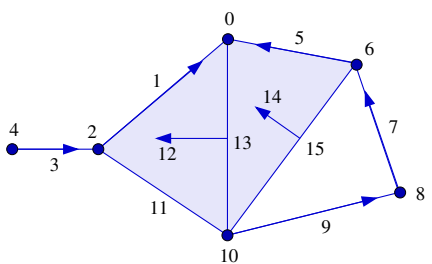

(b)

Fig. 1. (a) Forman function $F$, and (b) the corresponding discrete gradient vector field $V_{F}$, on a 2D simplicial complex. Each simplex $\sigma$ is labelled by the value of $F$ at $\sigma$.

In the example in Figure1 (a), a Forman function $F$ defined on a 2D simplicial complex is illustrated. Each simplex is labelled by its function value. Vertex labelled 0 and edge labelled 11 are critical simplexes of $F$.

Forman theory can be introduced starting from a notion of a discrete vector field, which can be imagined as a collection of arrows, connecting a $p$-simplex of $\Sigma$ to an incident $(p+1)$-simplex, such that each simplex is a head or a tail of at most one arrow. A simplex is critical if it is neither the head nor the tail of any arrow. A discrete vector field $V$ is a Forman gradient vector field if there are no closed $V$-paths in $V$. More formally, a discrete vector field $V$ on a simplicial complex $\Sigma$ is a collection of pairs $(\sigma, \tau)$, such that

(1) $\sigma$ is a $p$-simplex, and $\tau$ is a $(p+1)$-simplex of $\Sigma$,

(2) $\sigma$ is a face of $\tau(\sigma<\tau)$, and

(3) each simplex of $\Sigma$ is in at most one pair of $V$.

A $V$-path is a sequence $\sigma_{0}, \tau_{0}, \sigma_{1}, \tau_{1}, \ldots, \sigma_{r+1}$ of $p$-simplexes $\sigma_{i}$ and $(p+1)$ simplexes $\tau_{j}, i=0, . ., r+1, j=0, . ., r$, such that $\left(\sigma_{i}, \tau_{i}\right) \in V, \tau_{i}>\sigma_{i+1}$, and $\sigma_{i} \neq \sigma_{i+1}$. A sequence $\sigma_{0}, \tau_{0}, \sigma_{1}, \tau_{1}, \ldots, \sigma_{r+1}, r>0$, is a closed path if it is a 
$V$-path, and $\sigma_{r+1}=\sigma_{0}$. A discrete vector field $V$ is called a discrete (Forman) gradient vector field if and only if there are no closed $V$-paths in $V$. A critical simplex of $V$ of index $p$ is a $p$-simplex $\sigma$ which does not appear in any pair of $V$. In other words, a simplex $\sigma$ is critical if $V(\sigma)=\emptyset$, and $\sigma \notin I m V$. A gradient vector field $V$ is a subgraph (a forest) of the (non-oriented) Hasse diagram of $\Sigma$.

There is a correspondence between Forman functions and Forman gradient vector fields. For each Forman function $F$, a Forman gradient vector field $V_{F}$ can be constructed, by drawing an arrow from a $p$-simplex $\sigma$ to a $(p+1)$-simplex $\tau$ (adding a pair $(\sigma, \tau)$ to $\left.V_{F}\right)$ if $\tau>\sigma$ and $F(\tau) \leq F(\sigma)$. Conversely, for each Forman gradient vector field $V$ there exists a (non-unique) Forman function $F$ such that the gradient field $V_{F}$ of $F$ is $V$ [7]. The example in Figure 1 (b) shows a Forman gradient vector field $V_{F}$ of the Forman function $F$ in Figure 1 (a).

\section{Related Work}

There have been several proposals which construct a Forman gradient vector field, or a Forman function, on a simplicial complex $\Sigma$. Computation of a Forman gradient vector field $V$ with minimal number of critical cells on a $2 \mathrm{D}$ simplicial complex $\Sigma$ with a manifold domain has been discussed by Lewiner et al. [12], with the objective to compute the homology of $\Sigma$. It is not possible to analyze a specific scalar field $f$ given on vertices of $\Sigma$ using this approach. Forman theory has been used to build approximations of a Morse-Smale complex by Cazals et al. [1], with the objective to segment the surface of a molecule, using a discrete Connolly function $f$ computed on each vertex of the surface.

In [11], King et al. consider the problem of computing a Forman gradient vector field $V$ and the corresponding Forman function $F$, which extends a scalar field $f$ given on the vertices of a triangulated surface. Simplification of $V$ by cancellation of critical simplexes is included in the algorithm. Forman function $F$ is arbitrarily close to the maximum of $f$ over vertices of $\sigma$. Forman function which we construct here can be easily converted into another Forman function $G$, satisfying the same condition. As opposed to the gradient field EGrad $f$ which we construct, gradient field $V$ constructed in [11] does not always point in the direction in which scalar field $f$ is decreasing. The approach in [11] is extended to arbitrary dimensions by Jerše and Mramor Kosta in [10], and it is used to define a Forman gradient vector field $V$ on a regular cell complex $\Gamma$ with a manifold carrier $\Delta \Gamma$. Descending regions related to critical cells of $V$ are defined. It is shown that after a finite number of subdivisions, all descending regions are topological disks. Ascending regions are defined using the dual complex.

In [9], Gyulassy et al. use Forman theory and a divide-and-conquer technique to compute an approximation of the Morse-Smale complex of a scalar field $f$ defined on the vertices of a regular cell complex $\Gamma$ with manifold carrier. It is not guaranteed that the constructed discrete gradient vector field $V$, if applied to a triangulated domain, points in the direction in which the scalar field $f$ is decreasing. Forman function $F$ corresponding to field $V$ is not constructed. 
In [3], Cousty et al. demonstrate a link between collapse operator for maps and watersheds on pseudomanifols. It is assumed that a function $F$ (which is not a Forman function) is defined on all simplexes of a pseudomanifold. A collapse is used on the level sets of $F$ to obtain watershed of $F$.

\section{The Smale-Like Discrete Decomposition}

In this Section, we describe a dimension independent region-based method, introduced in [4], for extracting an approximation of a descending Morse complex related to maxima, called a Smale-like decomposition, starting from a scalar field $f$ defined on the vertices of a triangulated manifold domain $\Sigma$.

The process of region-growing is based on the elevation values at the vertices of $\Sigma$, and the connectivity of $\Sigma$. It is assumed that $f(p) \neq f(q)$ if $p \neq q$ ( $p$ and $q$ are vertices of $\Sigma$ ). This condition, which is assumed in other methods that produce a segmentation of $\Sigma$ using Forman theory [9 11], ensures, over regular complexes, the uniqueness of the decomposition, and can be achieved by a small perturbation of scalar field $f$. The decomposition obtained will depend on the way the input data is perturbed. The algorithm extracts descending regions of a discrete Morse complex, related to maxima of $f$, and can be modified in an obvious way to obtain ascending regions related to minima. Overlay of ascending and descending regions defines an approximation of a discrete Morse-Smale complex.

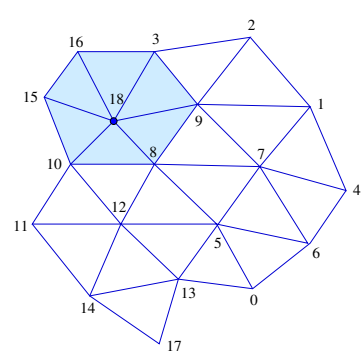

(a)

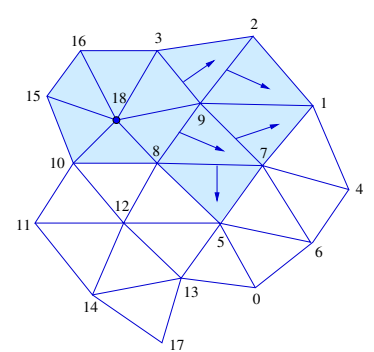

(b)

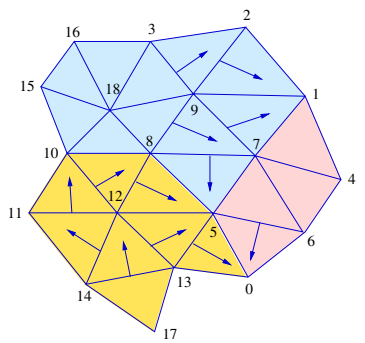

(c)

Fig. 2. (a) Initialization, and (b) the complete region associated with point $p$ at elevation 18. (c) The decomposition of a two-dimensional triangulated domain $D$. Points are labelled by their function value. Arrows indicate the region growing process.

In the preprocessing step, the vertices of $\Sigma$ are sorted in descending order of their elevation, and are processed in this order. A current set $K$ is kept, which is initialized to be equal to $\Sigma$. A descending region $C(p)$, associated with global maximum $p$, is initialized with all the simplexes in $\overline{S t}(p)$ (the closure of the star of $p$ in $K)$, and the boundary $\partial C(p)$ of $C$ is equal to the link $L k(p)$ in $K$. For example, in Figure 2 (a), each vertex $p$ of a $2 \mathrm{D}$ triangulated surface is labelled by 
the value of a scalar field $f$ at $p$. A global maximum of $f$ is achieved at a point labelled 18, and the descending region $C(18)$ is initialized with all the simplexes in the closed star of vertex 18 .

After the initialization step, the region growing step of the algorithm is performed. For each top $((n-1)$-dimensional $)$ simplex $\gamma \in \partial C(p)$, which is incident to another simplex (cone) $q * \gamma \in K-C(p)$, if $f(q)$ is less than $f(r)$, for all vertices $r$ of $\gamma$, then $C(p)$ is extended to $C(p) \cup \overline{q * \gamma}$, and the boundary $\partial C(p)$ of $C(p)$ is updated by replacing $\gamma$ with all faces of the cone $q * \gamma$ that contain $q$. Region growing is illustrated in Figure 2(b). Region $C(p)$ is extended iteratively, until no more simplexes can be added to it while maintaining the above property. Then, the interior of $C(p)$ is deleted from $K$, and the process is repeated until there are no more $n$-simplexes in $K$. After all vertices of the example illustrated in Figure 2 are processed, decomposition given in Figure 2 (c) is obtained. Figure 3 shows the results produced by the Smale-like decomposition algorithm on a 2D terrain data set, representing Mount Marcy (courtesy of USGS).

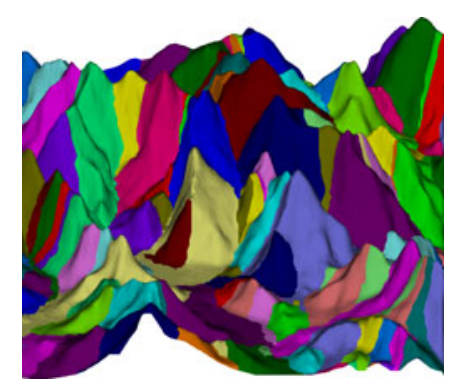

(a)

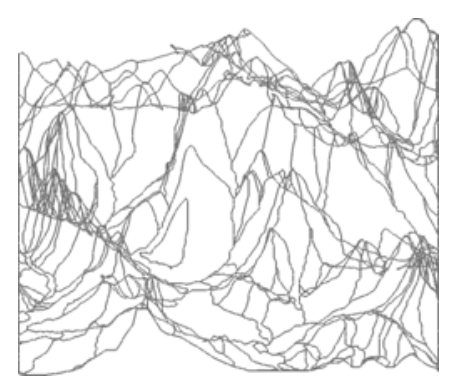

(b)

Fig. 3. (a) Perspective view of the 119 unstable components, produced by the Smalelike decomposition algorithm, applied to Mount Marcy with 69718 triangles. (b) Wireframe model.

\section{Extended Discrete Gradient Field}

A discrete gradient field Grad $f$ can be defined using the decomposition algorithm described in the previous Section. It associates with each $(n-1)$ dimensional simplex $\gamma$, which has been used in the extension process, the cone $q * \gamma$ added to $C(p)$, i.e., Grad $f(\gamma)=q * \gamma$. Here, we extend the field Grad $f$ into a discrete gradient vector field EGrad $f$, defined on all simplexes of $\Sigma$, which we call extended discrete gradient field.

Intuitively, for each pair $(\gamma, q * \gamma)$, such that Grad $f(\gamma)=q * \gamma$, EGrad $f$ can be defined over all $i$-faces $\sigma^{i}$ of $(n-1)$-simplex $\gamma, 0 \leq i \leq n-2$, by associating $\sigma^{i}$ with cone $q * \sigma^{i}$, i.e., by adding the pair $\left(\sigma^{i}, q * \sigma^{i}\right)$ to $E G r a d f$ (by setting EGrad $\left.f\left(\sigma^{i}\right):=q * \sigma^{i}\right)$. Geometrically, this extension consists of emanating vectors from all faces $\sigma^{i}$ of $\gamma$ towards vertex $q$. This is compatible with Smalelike decomposition process since $f(q)<f(r)$ for all vertices $r$ of $\gamma$. Note that, 
according to this construction, all faces of $\gamma$ are not critical since they are tails of vectors. If any of such $(n-1)$-dimensional cones $q * \sigma^{n-2}$ is used later to expand $C(p)$ in the Smale-like decomposition process, the pair $\left(\sigma^{n-2}, q * \sigma^{n-2}\right)$ has to be removed from EGrad $f$, and the $(n-1)$-dimensional cone $q * \sigma^{n-2}$ is processed in the same way as $(n-1)$-simplex $\gamma$ above. In this way we avoid having a simplex $\sigma$ such that there is an arrow starting at, and an arrow ending at $\sigma$. We now give a formal description of the step-by-step algorithm which defines a discrete field EGrad $f$. Steps (1), (2), and (3) define EGrad $f$ on $(n-1)$-dimensional, $i$-dimensional (for $0 \leq i \leq(n-3)$ ), and $(n-2)$-dimensional simplexes of $\Sigma$, respectively.

Definition 1. The extended discrete gradient field EGrad $f$ of a scalar function $f$ (and of the corresponding discrete gradient field Grad $f$ ) defined on the vertices of an n-dimensional triangulated domain $\Sigma$ is given by

(1) if $\gamma$ is an $(n-1)$-simplex such that Grad $f(\gamma)=q * \gamma(\gamma$ is expanding a component $C(p)$ to include the cone $q * \gamma)$ then EGrad $f(\gamma):=q * \gamma$.

(2) For all $i$-simplexes $\sigma^{i}<\gamma$, where EGrad $f(\gamma)=q * \gamma$ for some $q$, and $i=$ $0, \ldots, n-3$, EGrad $f\left(\sigma^{i}\right):=q * \sigma^{i}$ if EGrad $f\left(\sigma^{i}\right)$ has not been defined before when another $(n-1)$-simplex $\gamma$ incident to $\sigma^{i}$ was considered. Otherwise, $\sigma^{i}$ is skipped (since it has already an attached value by EGrad $f$ ).

(3) For all $(n-2)$-dimensional simplexes $\sigma^{n-2}<\gamma$, where EGrad $f(\gamma)=q * \gamma$ for some $q$, we distinguish two cases:

(a) If the $(n-1)$-dimensional cone $q * \sigma^{n-2}$ does not participate in the expansion process of $C(p)$, and if it is not already paired with some $(n-2)$ simplex in EGrad $f$ (if it is not in the image of EGrad $f$ ), then we set EGrad $f\left(\sigma^{n-2}\right):=q * \sigma^{n-2}$.

(b) Otherwise, we set (temporarily) EGrad $f\left(\sigma^{n-2}\right):=\emptyset$. In this case, cone $q * \sigma^{n-2}$ represents a new expanding $(n-1)$-simplex of $C(p)$. Return to steps (1), (2) and (3) to define EGrad $f$ on cone $q * \sigma^{n-2}$ (i.e., to set EGrad $f\left(q * \sigma^{n-2}\right):=$ Grad $\left.f\left(q * \sigma^{n-2}\right)\right)$, on $i$-faces of cone $q * \sigma^{n-2}$, $0 \leq i \leq n-3$, and on $(n-2)$-faces of cone $q * \sigma^{n-2}$, respectively.

For each simplex $\sigma$ in the open star of a vertex $p$ which starts a new component, such that $\sigma$ does not belong to the boundary or interior of another component $C(t), \sigma$ is a critical simplex, and EGrad $f(\sigma):=\emptyset$. We note here that our extended field EGrad $f$ depends on the order in which top simplexes are processed. Such order dependence is common to other approaches which compute a discrete gradient vector field starting from a scalar function $f$ [9, 11].

We present in Figure 4 the extended discrete gradient field EGrad $f$ induced by scalar field $f$, illustrated in Figure 2 , Simplexes $\gamma$ are edges and their faces $\sigma^{i}$ are vertices (i.e., we have only $\left.i=0\right)$. Edge $\gamma=[3 ; 9]$ expands the component $C(18)$, by adding triangle $[3 ; 9 ; 2]$ to $C(18)$, and EGrad $f([3 ; 9]):=[3 ; 9 ; 2]$ (step $(1))$. End points of edge $[3 ; 9]$ are the $(n-2)$-simplexes described above. Edge $[3 ; 2]$ does not participate in the expansion of the new component $C(18):=$ $C(18) \cup[3 ; 9 ; 2]$. Thus, EGrad $f([3])=[3 ; 2]$ (step $(3 \mathrm{a})$ ). The other vertex $[9]$ of edge $[9 ; 2]$, with vertex $[2]$ forms an edge that expands the updated $C(18)$, 


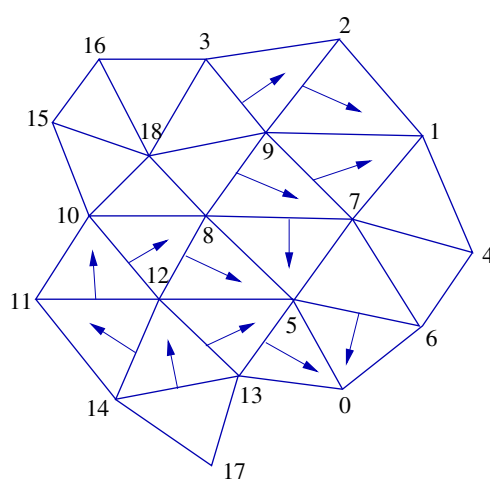

(a)

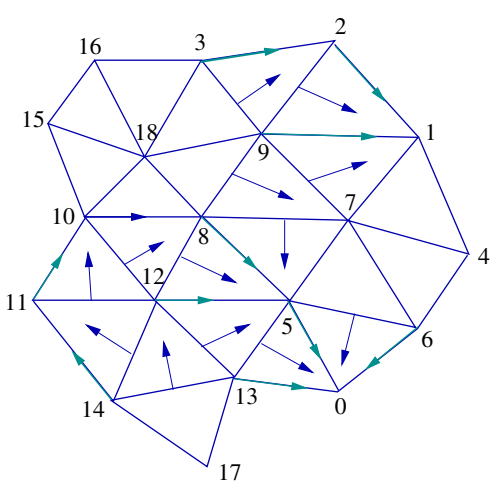

(b)

Fig. 4. (a) Function Grad $f$, defined by the Smale-like decomposition of Figure 2 (b) The corresponding extended discrete gradient field EGrad $f$.

so (temporarily) EGrad $f([9])=\emptyset$ (step (3b). Vertex [9] is visited again when triangle $[9 ; 2 ; 1]$ is considered. Function EGrad $f$ associates vertex [9] with edge $[9 ; 1]$, since edge $[9 ; 1]$ does not expand $C(p)$ (step $(3 \mathrm{a})$ ). Vertex $[9]$ is revisited again when triangle $[9 ; 1 ; 7]$ is considered. The process skips here vertex $[9]$ since it has already a non-empty value by EGrad $f$ (step (2)). We have here one (global) minimum [0] and the entire stars $S t(18)$ and $S t(17)$, and a part of $S t(7)$ as critical cells corresponding to local maxima 18 and 17.

Theorem 1. The extended discrete gradient field EGrad $f$ defined by the algorithm in Definition 1 is a Forman gradient vector field.

Proof. We need to show that (i) EGrad $f$ is a discrete vector field, and (ii) there are no closed EGrad $f$-paths in $\Sigma$.

( $i$ ) From the construction of EGrad $f$ it is clear that EGrad $f$ pairs an $i$ simplex $\sigma^{i}$ with an $(i+1)$-simplex (cone) $q * \sigma^{i}, 0 \leq i \leq n-1$, that simplex $\sigma^{i}$ is a face of the cone $q * \sigma^{i}$, and that each simplex is a head or a tail of at most one arrow. Thus, EGrad $f$ is a discrete vector field.

(ii) Let us assume that the sequence $\sigma_{0}, \tau_{0}, \sigma_{1}, \tau_{1}, \ldots, \sigma_{r}, \tau_{r}, \sigma_{0}$ of $i$-simplexes $\sigma_{k}$ and $(i+1)$-simplexes $\tau_{l}, k, l=0, . . r, r>0$, is a closed EGrad $f$-path in $\Sigma$. Then $\left(\sigma_{k}, \tau_{k}\right) \in \operatorname{EGrad} f$, (i.e., EGrad $\left.f\left(\sigma_{k}\right)=\tau_{k}\right), \tau_{k}>\sigma_{k+1}$, and $\sigma_{k} \neq \sigma_{k+1}$. By the construction of EGrad $f$, and the condition that $f(p) \neq f(q)$ for any two vertices $p$ and $q$ of $\Sigma$, we have that $\tau_{0}=q * \sigma_{0}$, and $f(q)<f(p)$, for each vertex $p$ of $\sigma_{0}$, implying that $\min _{p \in \tau_{0}} f(p)<\min _{p \in \sigma_{0}} f(p)$. Simplex $\sigma_{1}$ is a face of $\tau_{0}$ different from $\sigma_{0}$, which implies that $q$ is a vertex of $\sigma_{1}$, and $\min _{p \in \sigma_{1}} f(p)=\min _{p \in \tau_{0}} f(p)=f(q)$. Now $\min _{p \in \sigma_{1}} f(p)=\min _{p \in \tau_{0}} f(p)<\min _{p \in \sigma_{0}} f(p)$. If we continue in this way inductively, we conclude that $\min _{p \in \sigma_{0}} f(p)=\min _{p \in \tau_{r}} f(p)<\min _{p \in \sigma_{0}} f(p)$, a contradiction. Thus, there are no closed EGrad $f$-paths in $\Sigma$, and EGrad $f$ is a Forman gradient vector field. 


\section{A Discrete Forman Function for the Extended Gradient Field}

In this Section, we construct a Forman function $F$, which extends scalar field $f$, such that the discrete gradient vector field $V_{F}$ of $F$ coincides with $V$. We denote as $d(\gamma)$ the length of the longest $V$-path which starts at $\gamma$, as $D$ the maximum of $d(\gamma)$ over all cells of $\Sigma$, and as $A$ the minimum (absolute) difference of values of function $f$ over all vertices of $\Sigma$.

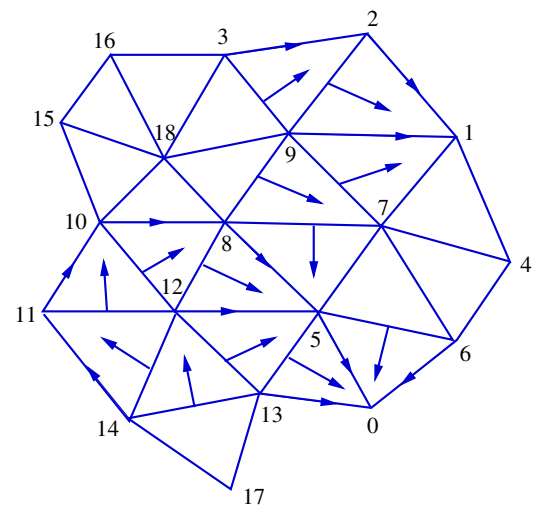

(a)

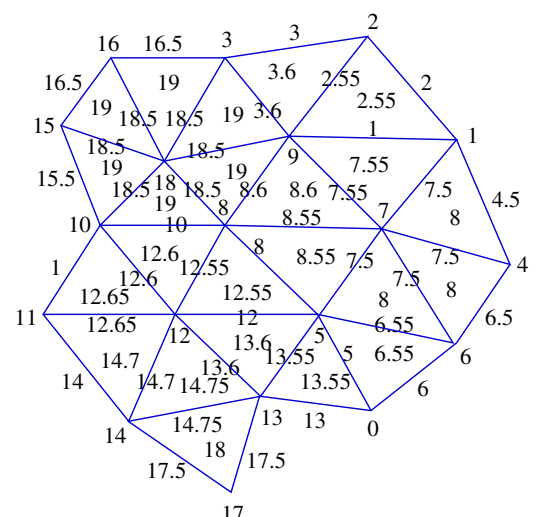

(b)

Fig. 5. Field EGrad $f$ (a) and the corresponding Forman function $F$ (b)

Let $\Sigma$ be a simplicial complex, and let $f$ be a scalar function defined on the vertices of $\Sigma$. Let $V$ be a discrete gradient vector field on $\Sigma$, consisting of pairs of simplexes $(\sigma, \tau)$, i.e., such that $V(\sigma)=\tau=q * \sigma$, where $q$ is a vertex of $\Sigma$. If $f(q)<f(p)$ for all vertices $p \in \sigma$, then an extended discrete function $F$ on all $i$-simplexes $\gamma$ of $\Sigma, 0 \leq i \leq n$, given by

(1) If $\gamma$ is critical, then $F(\gamma)=\max _{p \in \gamma} f(p)+i \frac{A}{n}$,

(2) If $V(\gamma) \neq \emptyset$, then $F(\gamma)=\max _{p \in \gamma} f(p)+i \frac{A}{n}\left(1+\frac{d(\gamma)}{n D}\right)=\max _{p \in \gamma} f(p)+i \frac{A}{n}+i \frac{A}{n^{2}} \frac{d(\gamma)}{D}$, (3) If $\gamma=V(\sigma)$, then $F(\gamma)=F(\sigma)$,

is a Forman function, such that gradient vector field $V_{F}$ of $F$ coincides with $V$. For $i=0$, an $i$-dimensional simplex is a vertex $p$ of $\Sigma$, for which we have $F(p)=f(p)$. Thus, $f$ is the restriction of $F$ on the set of vertices of $\Sigma$. The first term, $\max _{p \in \gamma} f(p)$, relates functions $f$ and $F$. The second term, $i \frac{A}{n}$, ensures that each face $\tau$ of a simplex $\sigma$ has a lower $F$ value than $\sigma$. The third term, $i \frac{A}{n^{2}} \frac{d(\gamma)}{D}$, ensures that function $F$ is non-increasing along each descending gradient path. In Figure 5, we give an example of an extended discrete function $F$, induced by the extended discrete field of Section [6. Here, $n=2, D=5$, and $A=1$.

In the proof that $F$ is a Forman function, we use the following facts: 
(a) if $\sigma$ is a face of $\tau$, then $\max _{p \in \sigma} f(p) \leq \max _{p \in \tau} f(p)$,

(b) if $\sigma$ is an $i$-dimensional simplex, and $\tau$ is a $j$-dimensional simplex of $\Sigma$ $(0 \leq i<j \leq n)$, then

$$
i \frac{A}{n} \leq i \frac{A}{n}+i \frac{A}{n^{2}} \frac{d(\sigma)}{D} \leq i \frac{A}{n}+i \frac{A}{n^{2}}<i \frac{A}{n}+n \frac{A}{n^{2}}=i \frac{A}{n}+\frac{A}{n}=(i+1) \frac{A}{n} \leq j \frac{A}{n} .
$$

We consider a pair of simplexes $\sigma$ and $\tau$, such that $\sigma$ is an $i$-simplex, $\tau$ is a $j$-simplex, $0 \leq i<j \leq n$, and $\sigma$ is a (proper) face of $\tau$, and we show that $F(\sigma)<F(\tau)$ if $V(\sigma) \neq \tau$. Then, the conclusion will follow for $i=j-1$. We consider separately the cases when $\sigma$ and $\tau$ belong to one of the three sets which partition the set of all simplexes of $\Sigma$, namely critical simplexes, simplexes which have a non-empty image by $V$, and simplexes which are in the image of $V$. For lack of space, we give the proof in two cases only.

(i) $\sigma$ is critical, and $V(\tau) \neq \emptyset$. Then by (a) and (b)

$$
F(\sigma)=\max _{p \in \sigma} f(p)+i \frac{A}{n}<\max _{p \in \tau} f(p)+j \frac{A}{n}+j \frac{A}{n^{2}} \frac{d(\tau)}{D}=F(\tau) .
$$

(ii) $V(\sigma) \neq \emptyset$, and $\tau \in \operatorname{Im} V$.

If $V(\sigma)=\tau$, then $F(\sigma)=F(\tau)$.

If $V(\sigma)=\gamma \neq \emptyset$ and $\tau=V\left(\tau^{\prime}\right)$ for some $\tau^{\prime} \neq \sigma$, then $F(\tau)=F\left(\tau^{\prime}\right), \tau=q * \tau^{\prime}$ for some vertex $q \in \Sigma$.

If $\tau^{\prime}$ is a $(j-1)$-dimensional co-face of $\sigma$ (if $\left.q \notin \sigma\right)$, then $j-1>i(\tau \neq V(\sigma)$, thus $\tau^{\prime} \neq \sigma$, and $\tau^{\prime}$ is a proper co-face of $\sigma$ ), and by (a) and (b)

$F(\sigma)=\max _{p \in \sigma} f(p)+i \frac{A}{n}+i \frac{A}{n^{2}} \frac{d(\sigma)}{D}<\max _{p \in \tau^{\prime}} f(p)+(j-1) \frac{A}{n}+(j-1) \frac{A}{n^{2}} \frac{d\left(\tau^{\prime}\right)}{D}=F(\tau)$.

If, on the other hand, $\tau^{\prime}$ is not a co-face of $\sigma$ (if $q \in \sigma$ ), then $f(q)<f(p)$, for all $p \in \tau^{\prime}$, and all other vertices of $\sigma$ different from $q$ (if they exist), are in $\tau^{\prime}$. Thus, $\max _{p \in \sigma} f(p) \leq \max _{p \in \tau^{\prime}} f(p)$.

If $j-1>i$, then by (b)

$F(\sigma)=\max _{p \in \sigma} f(p)+i \frac{A}{n}+i \frac{A}{n^{2}} \frac{d(\sigma)}{D}<\max _{p \in \tau^{\prime}} f(p)+(j-1) \frac{A}{n}+(j-1) \frac{A}{n^{2}} \frac{d\left(\tau^{\prime}\right)}{D}=F(\tau)$.

If $j-1=i$, then $\tau^{\prime}$ and $\sigma$ are $i$-cells, $V\left(\tau^{\prime}\right)=\tau$, and $\sigma$ is a face of $\tau$, implying that $d\left(\tau^{\prime}\right)=d(\sigma)+1$, and

$F(\sigma)=\max _{p \in \sigma} f(p)+i \frac{A}{n}+i \frac{A}{n^{2}} \frac{d(\sigma)}{D}<\max _{p \in \tau^{\prime}} f(p)+(j-1) \frac{A}{n}+(j-1) \frac{A}{n^{2}} \frac{d\left(\tau^{\prime}\right)}{D}=F(\tau)$.

It can be proven in a similar way in all other cases that for each pair of simplexes $\sigma$ and $\tau$, where $\sigma$ is a face of $\tau, F(\sigma)<F(\tau)$, unless if $V(\sigma)=\tau$, in which case $F(\sigma)=F(\tau)$. Thus, $F$ is a Forman function on $\Sigma$.

Note that we can define another Forman function $G$ by 


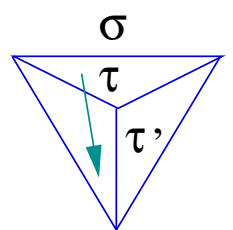

(a)

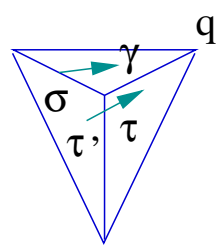

(b)

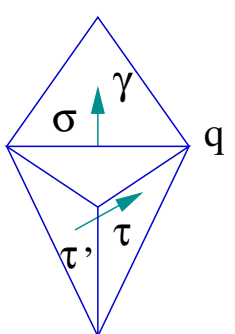

(c)

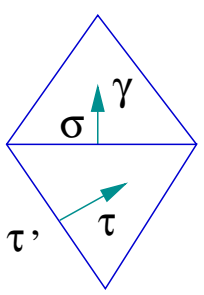

(d)

Fig. 6. (a) $\sigma$ is a critical simplex, $\tau$ is a co-face of $\sigma$, and $V(\tau) \neq \emptyset . V(\sigma)=\gamma \neq \emptyset$, and $\tau$ is a co-face of $\gamma$, (b) $\tau=V\left(\tau^{\prime}\right)$ and $\tau^{\prime}$ is a co-face of $\sigma ; \tau=V\left(\tau^{\prime}\right)$ and $\tau^{\prime}$ is not a co-face of $\sigma$ (with dimension of $\tau^{\prime}$ (c) greater than, or (d) equal to, dimension of $\sigma$ ).

(1) If $\gamma$ is critical, then $G(\gamma)=\max _{p \in \gamma} f(p)+i \frac{A}{n} \delta^{\prime}$,

(2) If $V(\gamma) \neq \emptyset$, then $F(\gamma)=\max _{p \in \gamma} f(p)+i \frac{A}{n} \delta^{\prime}+i \frac{A}{n^{2}} \frac{d(\gamma)}{D} \delta^{\prime \prime}$,

(3) If $\gamma=V(\sigma)$, then $F(\gamma)=F(\sigma)$,

where $\delta^{\prime}<\frac{\varepsilon}{2} \frac{1}{A}$, and $\delta^{\prime \prime}<\frac{\varepsilon}{2} \frac{n}{A}$ for some arbitrary small positive $\varepsilon<A$. Such function $G(\sigma)$ differs by at most $\varepsilon$ from the maximum of $f$ over all vertices of $\sigma$.

\section{Concluding Remarks}

We have defined an extended form EGrad $f$ of a discrete gradient vector field Grad $f$, and we have defined a Forman function $F$ which coincides with $f$ on the vertices of $\Sigma$, such that the gradient field of $F$ coincides with EGrad $f$. Our work has similarities with the work by King et al. [11], and by Gyulassy et al. [9] (when applied to a triangulated domain). The main difference is that our vector field always points in the direction of the vertex with smallest function value.

This property is not always satisfied by other approaches that construct a Forman gradient vector field $V$ starting from a scalar function $f$ given on vertices of $\Sigma$, as pointed out in [9, 11]. In this sense, our gradient field EGrad $f$ reflects better the behavior of the scalar field $f$.

Moreover, in [9], a Forman function whose gradient vector field coincides with $V$ is not constructed. In [11], such a Forman function $F$, which extends scalar field $f$, is constructed. It satisfies an additional condition that for each simplex $\sigma, F(\sigma)$ is arbitrarily close to the $\max _{p \in \sigma} f(p)$. We have shown in Section 7 that the Forman function $F$ that we construct can be re-scaled to another Forman function $G$ satisfying the same condition.

As an application of our work, we plan to apply the Forman simplification (cancellation) operator to our extended discrete gradient field EGrad $f$ and discrete gradient field Grad $f$. In this way, we hope to reduce significantly the number of critical cells in $\Sigma$. We plan to investigate how this simplification 
affects the vector fields, in order to define a multi-resolution model based on both Morse and Forman theory, and obtain a compact and topology preserving multi-resolution representation of scalar field $f$.

Acknowledgement. We would like to thank Emanuele Danovaro for Figure 3 . This work has been partially supported by the National Science Foundation under grant CCF-0541032.

\section{References}

1. Cazals, F., Chazal, F., Lewiner, T.: Molecular Shape Analysis Based upon the Morse-Smale Complex and the Connolly Function. In: Proceedings of the nineteenth Annual Symposium on Computational Geometry, pp. 351-360 (2003)

2. Comić, L., De Floriani, L.: Multi-Scale 3D Morse Complexes. In: International Conference on Computational Science and its Applications (ICCSA), Workshop on Computational Geometry and Applications, pp. 441-451 (2008)

3. Cousty, J., Bertrand, G., Couprie, M., Najman, L.: Collapses and Watersheds in Pseudomanifolds. In: Wiederhold, P., Barneva, R.P. (eds.) IWCIA 2009. LNCS, vol. 5852, pp. 397-410. Springer, Heidelberg (2009)

4. Danovaro, E., De Floriani, L., Mesmoudi, M.M.: Topological Analysis and Characterization of Discrete Scalar Fields. In: Asano, T., Klette, R., Ronse, C. (eds.) Geometry, Morphology, and Computational Imaging. LNCS, vol. 2616, pp. 386402. Springer, Heidelberg (2003)

5. Danovaro, E., De Floriani, L., Vitali, M., Magillo, P.: Multi-Scale Dual Morse Complexes for Representing Terrain Morphology. In: GIS 2007: Proceedings of the 15th Annual ACM International Symposium on Advances in Geographic Information Systems, pp. 1-8. ACM, New York (2007)

6. De Floriani, L., Mesmoudi, M.M., Danovaro, E.: Smale-Like Decomposition for Discrete Scalar Fields. In: Proceedings International Conference on Pattern Recognition, ICPR (2002)

7. Forman, R.: Combinatorial Vector Fields and Dynamical Systems. Mathematische Zeitschrift 228, 629-681 (1998)

8. Forman, R.: Morse Theory for Cell Complexes. Advances in Mathematics 134, 90-145 (1998)

9. Gyulassy, A., Bremer, P.T., Hamann, B., Pascucci, V.: A Practical Approach to Morse-Smale Complex Computation: Scalability and Generality. Transactions on Visualization and Computer Graphics 14(6), 1619-1626 (2008)

10. Jerše, G., Mramor Kosta, N.: Ascending and descending regions of a discrete morse function. Comput. Geom. Theory Appl. 42(6-7), 639-651 (2009)

11. King, H., Knudson, K., Mramor, N.: Generating Discrete Morse Functions from Point Data. Experimental Mathematics 14(4), 435-444 (2005)

12. Lewiner, T., Lopes, H., Tavares, G.: Applications of Forman's Discrete Morse Theory to Topology Visualization and Mesh Compression. Transactions on Visualization and Computer Graphich 10(5), 499-508 (2004)

13. Matsumoto, Y.: An Introduction to Morse Theory, Translations of Mathematical Monographs, vol. 208. American Mathematical Society, Providence (2002)

14. Milnor, J.: Morse Theory. Princeton University Press, New Jersey (1963) 